\title{
Research on Image Segmentation Algorithm Based on Entropy and PSO Algorithm
}

\author{
Lida Qiu*, Ping Fu and Tianjian Liu
}

Department of Physics \& Electronic Information Engineering, Minjiang University, Fuzhou, Fujian 350001, China

\begin{abstract}
Because image segmentation is the base of image identification, analysis and interpretation, the image segmentation has been widely used in many fields. And PSO (Particle Swarm Optimization) algorithm is one of the most common image segmentation algorithms. However, it has the problems of premature convergence and local optimum. To solve the problems, ISABEP (Image Segmentation Algorithm Based on Entropy and PSO) algorithm has been proposed. Simulation demonstrates that the proposed algorithm has fast convergence speed.
\end{abstract}

Keywords: Image segmentation, Particle swarm optimization, Entropy, Threshold.

\section{INTRODUCTION}

Under research of the image, people are only interested in some parts of the images, which is called target, and the rest is called background. According to the different characteristics of the image, image segmentation as a kind of target extraction technology, can divide the image into several areas, one or several of which are the targets [1]. The characteristics may be color, grayscale, texture, and so on. Meanwhile, the accuracy of image segmentation directly affects the effectiveness of the subsequent image processing, therefore, scholars and enterprises always pay close attention to the development and the use of image segmentation $[2,3]$. To meet different applications, many image segmentation algorithms are proposed. However, the most of them are only applicable to certain applications. Besides, there isn't a unified selection standard, which brings many problems to hinder the use of image segmentation.

At present, image segmentation algorithms can be divided mainly into three class: threshold algorithm, edge detection algorithm, splitting algorithm and merging algorithm [4]. Among them, threshold algorithm is the most used. The basic idea of the threshold algorithm is that it firstly selects an image grey-scale value as the threshold, and then every pixel in the image will be compared with the threshold, finally, the image can be divided into two areas. The selection of threshold impacts on the effect of the segmentation significantly. Therefore, a lot of studies focus on how to select the effective and robust threshold. PSO (Particle Swarm Optimization) algorithm as one of most used threshold segmentation has many advantages, such as uncomplicated, easy to control and fast convergence [5]. Hence, it has been widely used. However, it has the problems of premature convergence and local optimum. To solve the problems, this paper proposes ISABEP (Image Segmentation Algorithm Based on

*Address correspondence to these authors at the Department of Physics \& Electronic Information Engineering, Minjiang University, Fuzhou, Fujian 350001, China; Tel: +8613538850618; E-mail: qld.qq@163.com
Entropy and PSO) algorithm. The core idea of ISABEP is the use of the PSOBM (PSO Based on Morlet) algorithm and MFE(Maximum Fuzzy Entropy) algorithm [6] to improve the performance of the image segmentation.

\section{RELATED THEORIES}

\subsection{Concept of Image Segmentation}

The concept of image segmentation is [7]: if $\mathrm{R}$ represents an image, the segmentation of $\mathrm{R}$ can be considered that $\mathrm{R}$ is divided into $\mathrm{N}$ non-empty subsets, $R_{1}, R_{2}, \mathrm{~L}, R_{N}, N \geq 1$, and all subsets must meet the following conditions:

$$
\begin{aligned}
& \text { Condition 1: } R=R_{1} \cup R_{2} \cup \mathrm{L} \cup R_{N} ; \\
& \text { Condition 2: } R_{i} \cap R_{j}=\Phi, 1 \leq i, j \leq N, i \neq j ; \\
& \text { Condition 3: } P\left(R_{i}\right)=\text { True, } i=1,2, \mathrm{~L}, N ; \\
& \text { Condition 4: } P\left(R_{i} \cap R_{j}\right)=\text { False }, 1 \leq i, j \leq N, i \neq j ; \\
& \text { Condition 5: } R_{i}, i=1,2, \mathrm{~L}, N \text { is a connected region. }
\end{aligned}
$$

Condition 1 means that after the image segmentation, union of all subsets is equal to the original image, and every pixel in the original image belongs to one of the subsets. Condition 2 means that after the image segmentation, a pixel in the original image can only belongs to one subset. Condition 3 means that every subset shouldn't be a null set. Condition 4 means that any two subsets shouldn't overlap. Condition 5 means that any two pixels in the same subset can be connected.

\subsection{MFE Algorithm}

Suppose that $X$ is the collection of objects denoted by $x$, then the fuzzy set $\mathrm{A}$ in $X$ is a set of ordered pairs: $A=\left\{\left(x_{i}, u_{A}\left(x_{i}\right)\right) \mid x_{i} \in X\right\}, u_{A}\left(x_{i}\right)$ is the membership function of $x_{i}$ to A. Therefore, the fuzzy entropy $E(A)$ is [8]: 


$$
E(A)=\frac{1}{n \ln 2} \sum_{i} S\left(u_{A}\left(x_{i}\right)\right), i=1,2, \mathrm{~L}, n
$$

$S\left(u_{A}\left(x_{i}\right)\right)$ is the entropy function, and the value is given as follow:

$$
\begin{aligned}
& S\left(u_{A}\left(x_{i}\right)\right)=-u_{A}\left(x_{i}\right) \ln \left[u_{A}\left(x_{i}\right)\right] \\
& -\left[1-u_{A}\left(x_{i}\right)\right] \ln \left[1-u_{A}\left(x_{i}\right)\right]
\end{aligned}
$$

$\mathrm{X}$ is a gray-scale image which is made of $M^{*} N$ pixels. And the greatest gray value of $\mathrm{X}$ is $\mathrm{L} . x_{m n}$ is the gray value of pixel coordinates $(m, n)$. The fuzzy entropy of twodimensional image given can be calculated as follows:

$$
E(X)=\frac{1}{M N \ln 2} \sum_{m} \sum_{n} S\left(u_{A}\left(x_{m n}\right)\right)
$$

In the gray-scale histogram, the horizontal axis is the gray value and the vertical axis is the number of pixels $h(g)$, therefore, Eq. (3) can be written:

$$
E(X)=\frac{1}{M N \ln 2} \sum_{n} S\left(u_{A}(g)\right) h(g)
$$

If $u_{1}(g)$ and $u_{2}(g)$ are respectively the membership of target and the membership of background, according to $\mathrm{S}$ function belonging to fuzzy set theory, the values of $u_{1}(g)$ and $u_{2}(g)$ are:

$$
\begin{aligned}
& u_{1}(g)=\left\{\begin{array}{l}
0,0 \leq g \leq a \\
2\left|\frac{g-a}{c-a}\right|^{2}, a \leq g<1 \\
1-2\left|\frac{c-g}{c-a}\right|^{2}, t \leq g<c \\
1, c \leq g<L
\end{array}\right. \\
& u_{2}(g)=1-u_{1}(g)
\end{aligned}
$$

Where $[a, c]$ is the range of gray value, and $t=(a+c) / 2$. The entropy of Area A and Area B is :

$$
\begin{aligned}
& E(A)=\frac{1}{\ln 2} \sum_{g=0}^{t-1} S\left(u_{1}(g)\right) h(g) \\
& E(B)=\frac{1}{\ln 2} \sum_{g=t}^{L-1} S\left(u_{2}(g)\right) h(g)
\end{aligned}
$$

The fuzzy entropy of image is:

$$
E(X)=\frac{1}{\operatorname{Sum}(X)}(E(A)+E(B))
$$

Where $\operatorname{Sum}(\mathrm{X})$ is the sum of pixels of the image $\mathrm{X}$.

\subsection{PSOBM Algorithm}

In 1995, PSO algorithm was proposed by Kennedy. PSO is an iterative algorithm, which stems from the simulation of birds flock's looking for foods [9, 10]. The workflow of PSO is that: firstly, it chooses a set of particles as the initial population, and every particle is a feasible solution of the optimization problem. Secondly, every particle moves towards the optimal particle. Lastly, through many iterations, the rest of the particle is chosen as the solution of the optimization problem.

The mathematical description of PSO is: global optimization problem can be defined as $(P): \min \left\{f(x): x \in \Omega \subseteq R^{n}\right\}$ , $f: \Omega \subseteq R^{n} \rightarrow R^{l}$, where $(P)$ is the set of feasible solutions. The number of $(P)$ is called scale, and the member of $(P)$ is called particle. The speed and position of the i-th particle are respectively $V_{i}=\left(v_{i 1}, v_{i 2}, \mathrm{~L}, v_{i n}\right)^{T} \in \Omega$, $X_{i}=\left(x_{i 1}, x_{i 2}, \mathrm{~L}, x_{i n}\right)^{T} \in \Omega$. In the movement of the particles, $P_{i}=\left(p_{i 1}, p_{i 2}, \mathrm{~L}, p_{i M}\right)$ is used to record the optimum position of the $\mathrm{i}$-th particle. Index number $\mathrm{g}$ is used to mark the optimum position of the whole particles. The updates of the speed and position of every particle separately follow Eq. (8) and Eq. (9):

$$
\begin{gathered}
x_{i j}(t+1)=x_{i j}(t)+v_{i j}(t+1) \\
v_{i j}(t+1)=w v_{i j}(t)+c_{1} r a n d_{1}\left(p_{i j}(t)-x_{i j}(t)\right) \\
+c_{2} \operatorname{rand}_{2}\left(p_{g j}(t)-x_{i j}(t)\right)
\end{gathered}
$$

Where $i=1,2, \mathrm{~L}, M, d=1,2, \mathrm{~L}, n$, rand $_{1}$ and rand $_{2}$ are random numbers which follow $U(0,1) . c_{1}$ and $c_{2}$ are nonnegative constant coefficients, and $c_{1}, c_{2} \in[0,2] . w$ is the inertia coefficient.

Although PSO has many advantages, such as uncomplicated, easy to control and fast convergence, it still has obvious drawbacks of premature convergence and local optimum. To overcome the shortage of traditional PSO, people propose many improved algorithms. The essence of these algorithms is only the static adjustment of $w$ or the change of the particle. However the PSOBM can not only dynamically adjust the value of $w$ but also change some particles.

In PSOBM, the value of $w$ is:

$$
\omega=\omega_{0} \times\left(1-\left(\text { dist }_{i} / \text { dist }_{\max }\right)\right)
$$

Where $\omega_{0}$ is a random number which follows $\mathrm{U}(0.5,1)$, dist $_{i}$ is distance between the current particle and the global optimal value, $\operatorname{dist}_{i}=\left(\sum_{j=1}^{n}\left(p_{g j}-x_{i j}\right)\right)^{1 / 2}$, and $\operatorname{dist}_{\max }=\max \left(\operatorname{dist}_{i}\right)$, $i=1,2$,L , $M$. Adjusting the value of $w$ can make sure that the particle is always around the global optimal value, which can effectively avoid the occurrence of the premature convergence. To further improve the performance, the Morlet conversion is used for the particle with the probability $p_{m} \in[0,1]$ in PSOBM. The formula of the Morlet conversion is :

$$
\operatorname{mut}\left(x_{i j}(t)\right)=\left\{\begin{array}{l}
x_{i j}(t)+\sigma \times\left(x_{\max }-x_{i j}(t)\right), \sigma>0 \\
x_{i j}(t)+\sigma \times\left(x_{i j}(t)-x_{\min }\right), \sigma \leq 0
\end{array}\right.
$$


Where $\operatorname{mut}\left(x_{i j}(t)\right)$ is the new position of $x_{i, j}(t), x_{\min }$ and $x_{\max }$ are respectively the minimum value of $\mathrm{x}$ and the maximum value of $\mathrm{x}$, the value of $\sigma$ is given as follows:

$$
\sigma=\frac{1}{\sqrt{a}} e^{-(\phi / a)^{2}} / 2 \cos (5(\phi / a))
$$

Where, $\sigma=\frac{1}{\sqrt{a}} e^{-(\phi / a)^{2}} / 2 \cos (5(\phi / a))$, and the $a$ is:

$$
a=e^{-\ln (g)\left(1-t / t_{\max }\right)^{\xi^{w} w m}+\ln (g)}
$$

Where $g$ is the upper boundary of $a, \xi w m$ is the shape parameter of monotonic increasing equation, $t$ is the current iterations, and $t_{\text {max }}$ is the maximum current iterations.

\section{ISABEP ALGORITHM}

In order to further improve the image segmentation result, this paper proposes ISABEP algorithm. In ISABEP algorithm, the fuzzy entropy is introduced into PSOBE. According to the fuzzy theory, an image is equivalent to a fuzzy event, therefore, the image can be divided into two fuzzy sets $\mathrm{d}$ and $\mathrm{b}$ by threshold $\mathrm{T}$. Suppose that $\mu_{d}(k)$ and $\mu_{b}(k)$ are respectively the membership function of $\mathrm{d}$ and the membership function of $\mathrm{b} . H_{d}$ and $H_{b}$ are respectively the entropy of $d$ and the entropy of $b$. Their value can be calculated by the following equations:

$$
\begin{gathered}
\mu_{d}(k)\left\{\begin{array}{l}
1, k \leq a ; \\
1-\frac{(k-a)^{2}}{(c-a) \times(b-a)}, \quad a<k \leq b \\
\frac{(k-c)^{2}}{(c-a) \times(c-b)}, \quad b<k \leq c \\
0, \quad c<k
\end{array}\right. \\
\mu_{b}(k)\left\{\begin{array}{l}
0, k \leq a \\
\frac{(k-a)^{2}}{(c-a) \times(b-a)}, \quad a<k \leq b \\
1-\frac{(k-c)^{2}}{(c-a) \times(c-b)}, \quad b<k \leq c \\
1, \quad c<k
\end{array}\right. \\
H_{d}=\sum_{k=0}^{255} \frac{p_{k} \mu_{d}(k)}{p_{d}} \ln \left(\frac{p_{k} \mu_{d}(k)}{p_{d}}\right) \\
H_{b}=\sum_{k=0}^{255} \frac{p_{k} \mu_{b}(k)}{p_{b}} \ln \left(\frac{p_{k} \mu_{b}(k)}{p_{b}}\right)
\end{gathered}
$$

Where $0 \leq a<b<c \leq 225, b=(a+c) / 2, p_{k}=n_{k} /(m \times n)$, and $n_{k}$ is the number of elements in $D_{K}$, therefore the total fuzzy entropy of the fuzzy event is:

$$
H(a, c)=H_{d}+H_{b}
$$

According to the entropy theory, when the total fuzzy entropy of the fuzzy event is the maximum, the target can be effectively separated from the background. Suppose that when $(a, c)=\left(a_{\text {opt }}, c_{\text {opt }}\right), H(a, c)$ reaches the maximum, so the optimal threshold is:

$$
T_{\text {opt }}=b_{\text {opt }}=\left(a_{o p t}+c_{o p t}\right) / 2
$$

The details of ISABEP are described as follows.

(1) Initialization. Suppose that $\mathrm{N}$ is the number of particle swarm, $\mathrm{V}$ is the speed matrix of particle swarm, $\mathrm{X}$ is the position matrix, and $\mathrm{D}$ is the dimension. Initialization of $\mathrm{N}$, $\mathrm{V}, \mathrm{X}$ and $\mathrm{D}$ are done.

$$
\begin{gathered}
x_{i j}=x_{\min }+\left(x_{\max }-x_{\min }\right) \times \text { rand } \\
X=\left|\begin{array}{cc}
x_{11} & x_{12} \\
x_{21} & x_{22} \\
\mathrm{M} & \mathrm{M} \\
x_{N 1} & x_{N 2}
\end{array}\right| \\
v_{i j}=-v_{\max }+2 v_{\max } \times \text { rand } \\
V=\left|\begin{array}{cc}
v_{11} & v_{12} \\
v_{21} & v_{22} \\
\mathrm{M} & \mathrm{M} \\
v_{N 1} & v_{N 2}
\end{array}\right|
\end{gathered}
$$

Where rand is the random number, which follows $U(0,1)$, $v_{\max }$ is the maximum of $\mathrm{v}, x_{\max }$ and $x_{\min }$ are respectively the maximum of $\mathrm{x}$ and the minimum of $\mathrm{x}$. In general, $x_{\max }=L_{\max }, x_{\min }=L_{\min }+1, L_{\min }$ and $L_{\max }$ are respectively the minimum of the image and the maximum of the image.

(2) The fuzzy entropy is calculated by Eq. (18), then select the current optimal position of $P_{i}$ and the optimal position of the total particle set $P_{g}$.

(3) According to the above formulas, the value of $w$ is calculated by Eq. (10).

(4) The position and speed of every particle are calculated by Eq. (8) and Eq. (9). 
(5) Select the particles by probability $p_{m}$, and the selected particle carries through the Morlet conversion on the basis of Eq. (11).

(6) If the iterations reach the maximum times, go to step 7. Otherwise, go to step 2.

(7) The threshold $T_{\text {opt }}$ is calculated by the selection $(a, c)$, and then $T_{\text {opt }}$ is used to carry out the image segmentation.

\section{SIMULATION RESULTS}

To verify the effectiveness and efficiency of ISABEP proposed in this paper, we conduct extensive simulations for performance evaluation. We compare the performances of traditional PSO, PSOBM and ISABEP on the segmentation effectiveness. The images of Lena, Camel and Cinder are used in the experiment, as shown in Fig. (1). The three images have the different characteristics. Lena, Camel and Cinder are respectively multimodality, bimodality and unimodality, and the histograms of them are as shown in Fig. (2).

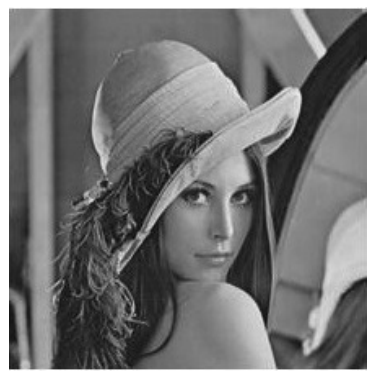

(a) Lena

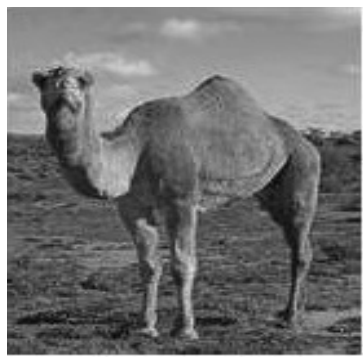

(b) Camel

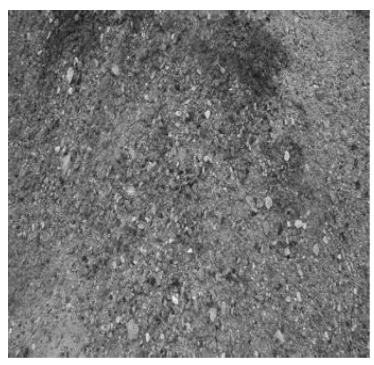

(c) Cinder

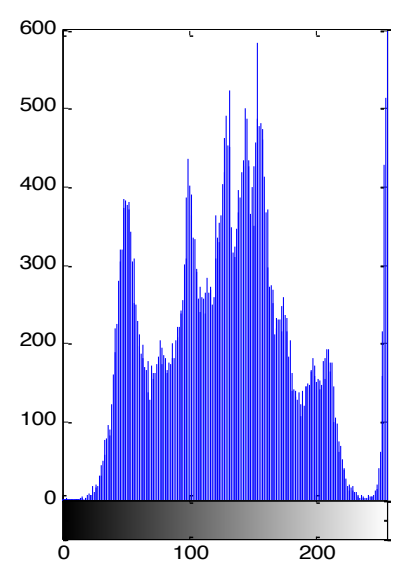

(a) Lena

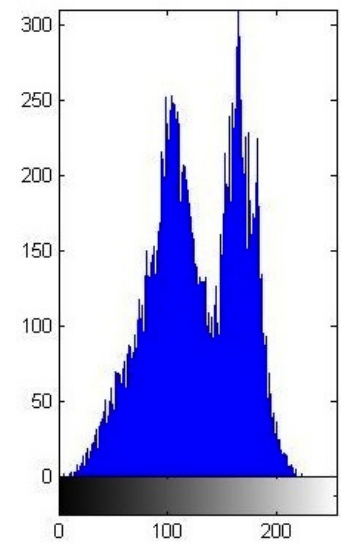

(b) Camel

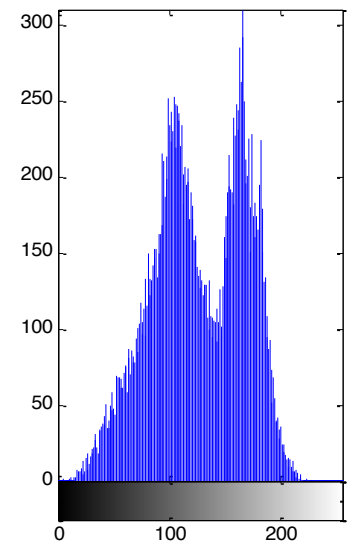

(c) Cinder

Fig. (2). Histogram.

The simulation parameters are set up as follows:

$$
\begin{aligned}
& \mathrm{N}=10 \\
& \mathrm{D}=2 \\
& \operatorname{tmax}=100 \\
& \mathrm{wmax}=0.9 \\
& \mathrm{wmin}=0.4 \\
& \mathrm{c} 1=\mathrm{c} 2=1.49 \\
& \mathrm{pm}=0.5 \\
& \mathrm{~g}=1000 \\
& \xi_{w m}=2
\end{aligned}
$$




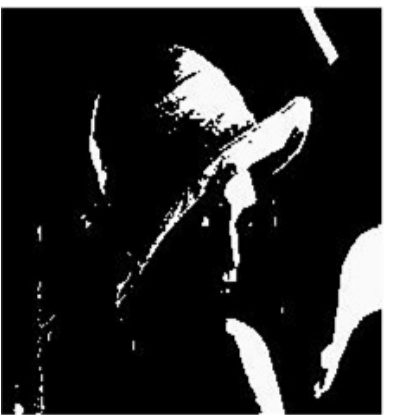

(a1)

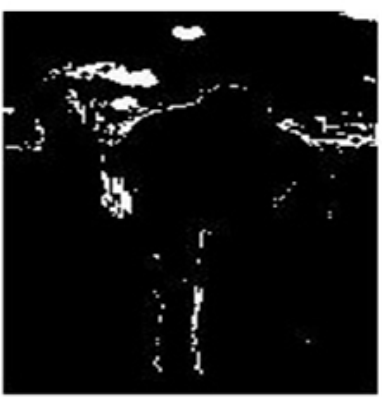

(a2)

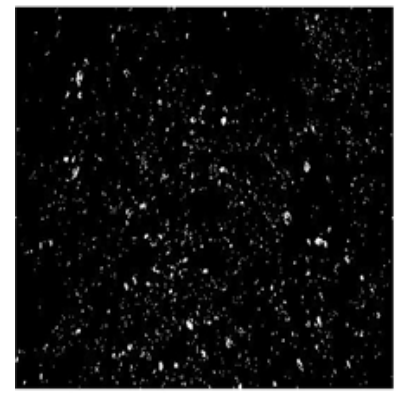

(a3)

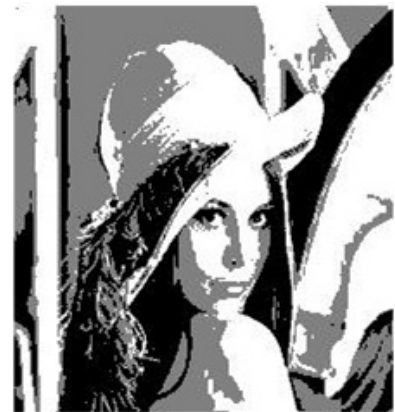

(b1)

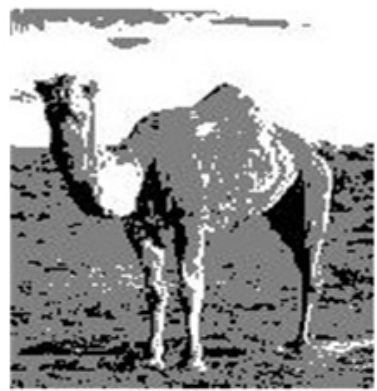

(b2)

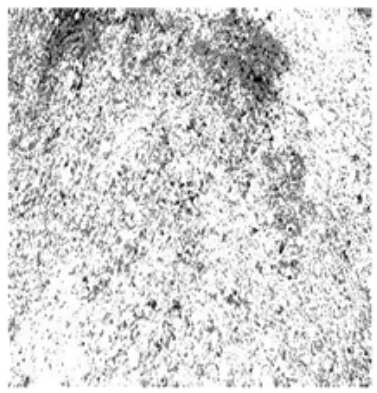

(b3)

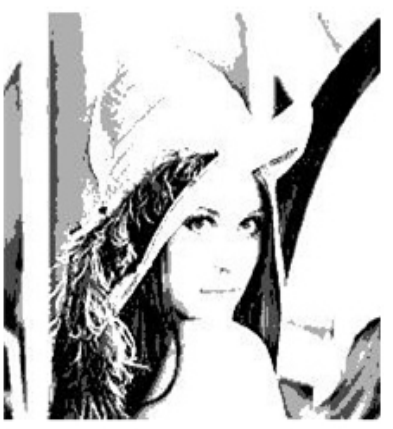

(c1)

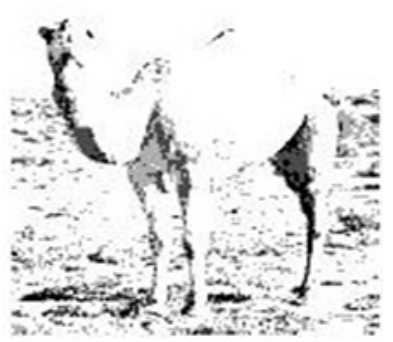

(c2)

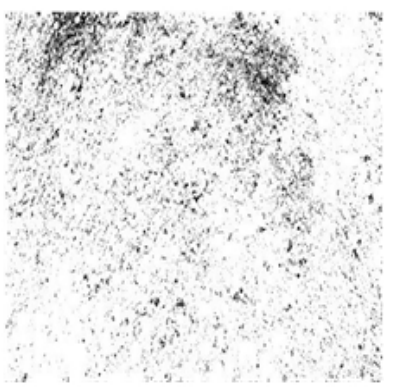

(c3)

Fig. (3). Segmentation effectiveness.

Fig. (3) gives the segmentation effectiveness. a1 a3 is the segmentation effectiveness of PSO, b1 b3 is the segmentation effectiveness of PSOBM, and $\mathrm{c} 1 \sim 3$ is the segmentation effectiveness of ISABEP. From Fig. (3), we can see that the performance of ISABEP is the best.

\section{CONCLUSION}

To solve the premature convergence and local optimum of PSO, we propose ISABEP. ISABEP uses PSOBM algorithm and MFE algorithm to improve the performance of the image segmentation. And simulations have verified the effectiveness of ISABEP.

\section{CONFLICT OF INTEREST}

The authors confirm that this article content has no conflict of interest.

\section{ACKNOWLEDGEMENTS}

The work was supported by The General Program of the National Natural Science Foundation of China
(No.51277091); The Key Program of Science and Technology Planning Project of Fujian Province of China (No. 2011H0017); The Key Program of Educational Commission of Fujian Province of China (No.JA12263); Program of Science and Technology Planning Project of Fuzhou of China (No.2013-G-86).

\section{REFERENCES}

[1] H. H. Cai, Y. Cheng, and B. X. Liu, "Ceramic microstructure image segmentation by mean shift," Applied Mechanics and Materials, vol. 423, pp. 2602-2605, 2013.

[2] N. R. Pal, and S. K. Pal, "A review on image segmentation techniques," Pattern recognition, vol. 26, pp. 1277-1294, 1993.

[3] S. Thilagamani, "A survey on image segmentation through clustering," International Journal of Research and Reviews in Information Sciences, vol. 1, pp. 120-126, 2011.

[4] B. Peng, L. Zhang, and D. Zhang, "A survey of graph theoretical approaches to image segmentation," Pattern Recognition, vol.46, pp. 1020-1038, 2013.

[5] R. V. Kulkarni, and G. K. Venayagamoorthy, "Particle swarm optimization in wireless-sensor networks: A brief survey," Systems, Man, and Cybernetics, Part C: Applications and Reviews, IEEE Transactions on, vol. 41, pp. 262-276, 2011. 
[6] J. Lan, and Y. Zeng, "Multi-threshold image segmentation using maximum fuzzy entropy based on a new 2D histogram," OptikInternational Journal for Light and Electron Optics, vol. 124, pp.3756-3760, 2013.

[7] J. Shi, and J. Malik, "Normalized cuts and image segmentation," Pattern Analysis and Machine Intelligence, IEEE Transactions on, vol. 22, pp. 888-905, 2000.
[8] H. J. Zimmermann, "Fuzzy set theory and its applications $2^{\text {nd }}$ revised ed.," Kluwer Academic Publishers, 1992.

[9] J. Kennedy, and R. C. Eberhart, "Particle swarm optimization," Proc. IEEE International Conference on Neural Networks, Piscataway NJ: IEEE Service Center, pp. 1942-1948, 1995.

[10] R. C. Eberhart, and Y. Shi, "Particle swarm optimization: developments, applications and resources," Evolutionary Computation, Proceedings of the 2001 Congress on. vol. 1, IEEE, 2001.

Received: January 05, 2015

Revised: April 10,2015

Accepted: May 12, 2015

(C) Qiu et al.; Licensee Bentham Open.

This is an open access article licensed under the terms of the Creative Commons Attribution Non-Commercial License (http://creativecommons.org/licenses/by-nc/4.0/) which permits unrestricted, non-commercial use, distribution and reproduction in any medium, provided the work is properly cited. 